\title{
Early recognition and appropriate pharmacotherapy for mixed depression: the key to resolving complex or treatment-refractory clinical cases
}

\author{
Minoru Takeshima, M.D. and Ph.D., ${ }^{1,2}$ \\ ${ }^{1}$ Meishinkai Shibata Hospital, Takaoka City, Japan \\ ${ }^{2}$ Department of Psychiatry, Tokyo Medical University, Tokyo, Japan
}

\begin{abstract}
Mixed depression refers to the co-existence of a major depressive episode (MDE) and sub-threshold manic/ hypomanic symptoms. Mixed depression is a common clinical entity, occurring in approximately $50 \%$ and $30 \%$ of MDEs due to bipolar disorder (BD) and major depressive disorder (MDD), respectively. However, it remains underdiagnosed and very often misdiagnosed as simply "major depression," adjustment disorder, anxiety disorder, or borderline personality disorder. Mixed depression suggests a diagnosis of BD in patients experiencing an MDE, and is associated with future progression to $\mathrm{BD}$, antidepressant-induced mania/hypomania, and a family history of BD in patients with MDD. Patients with mixed depression exhibit poor prognosis, experiencing more severe episodes of longer duration, less inter-episodic remission, higher recurrence rates, more rapid cycling, a higher rate of co-morbid substance use and anxiety disorders, and a higher risk of suicidality. Antidepressant use can exacerbate symptoms of agitation and irritability and induce newlydeveloped suicidality without improving depressive symptoms. Because treatment strategies differ for mixed and non-mixed states, it is important to screen all patients with depression for co-existing manic/hypomanic symptoms. In this report, the author briefly reviews the clinical presentation, diagnosis, and impact of mixed depression on the course of mood disorders and pharmacological treatment.
\end{abstract}

\section{Keywords: mixed depression, mixed features, bipolar disorder, depression, pharmacotherapy, treatment}

Received July 31, 2019 / Accepted July 31, 2019 / Published September 2, 2019.

\section{Introduction}

The term "mixed state" refers to the co-existence of manic and depressive symptoms. Descriptions of mixed states date back to the $1^{\text {st }}$ century, although the concept was first systematically evaluated by Emil Kraepelin in the early $20^{\text {th }}$ century [1]. Kraeplin argued that affective episodes involve weakness or excitement of the three domains of psychic life (mood, thinking, volition). In his view, a mixed state is indicated when at least one of these three domains is in opposition to the others (i.e., 6/8 combinations) [1]. In his eighth textbook, Kraeplin noted that patients with manic-depressive illnesses exhibit mixed states for most of the disease course [2]. However, his concept of mixed states was not widely accepted and was not incorporated into major psychiatric diagnostic systems such as the Diagnostic and Statistical Manual of Mental Disorders, Fourth Edition, Text Revision (DSM-IV-TR) [3]. In the DSM-IV-TR, a mixed episode is quite narrowly defined as the co-occurrence of DSM-IV-TRdefined mania and depression in the context of bipolar I disorder (BD-I).

Since the 1990s, many researchers have proposed 


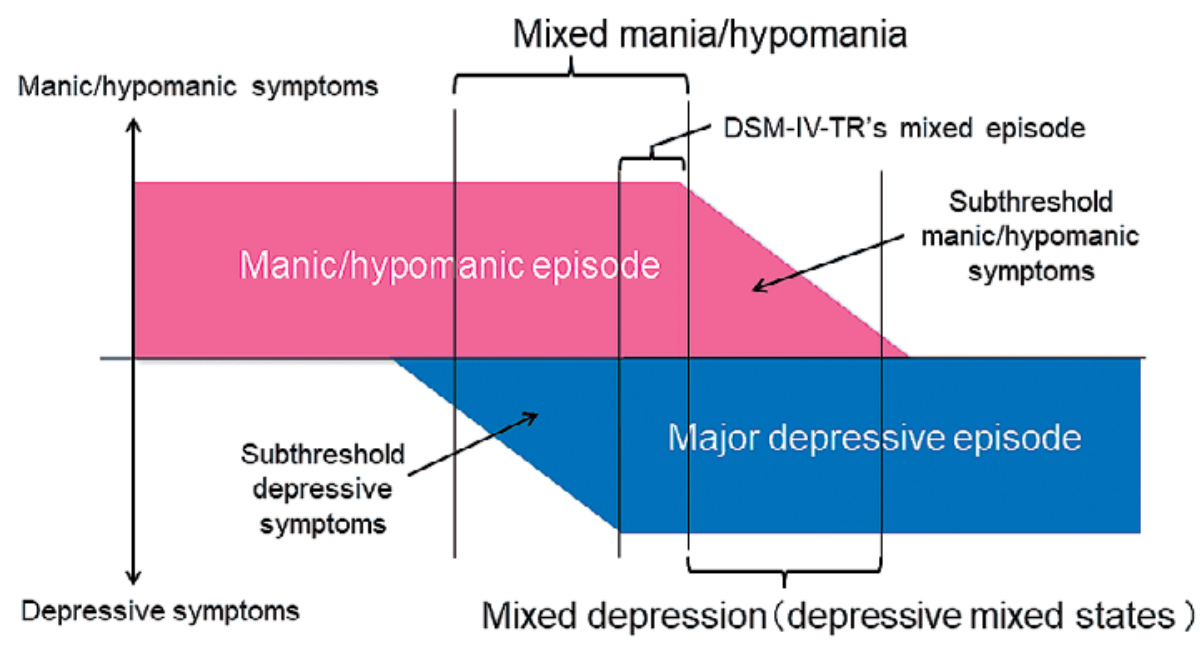

Figure 1. Schematic representation of the spectrum of mixed states. This figure is based on a 2017 report by Takeshima [4] (CNS Spectr). Abbreviations: DSM-IV-TR, Diagnostic and Statistical Manual of Mental Disorders, Fourth Edition, Text Revision.

redefining mixed states along a spectrum. Patients with manic or hypomanic episodes (ME/HME) experiencing sub-threshold depressive symptoms (mixed mania/hypomania) are at one end of this spectrum, followed by those experiencing full mixed states (corresponding to DSM-IV-TR-defined mixed episodes). Patients experiencing major depressive episodes (MDEs) with sub-threshold manic/hypomanic symptoms (mixed depression or depressive mixed state) lie at the other end of this spectrum [4]. A schematic representation of the spectrum of mixed states is shown in Figure 1, based on a 2017 report by Takeshima [4]. Recent studies have revealed the significance of this spectrum in relation to the diagnosis, prognosis, and treatment of mood disorders among patients experiencing mixed states [5]. Given this evidence, the narrow definition of "mixed episode" has been removed from the DSM-5, and the specifier "with mixed features" (i.e., at least three non-overlapping symptoms from the opposite pole) has been included for patients with BD-I and bipolar II disorder (BD-II) experiencing MEs/HMEs and for patients with BD-I, BD-II, and major depressive disorder (MDD) experiencing MDEs [6].

While the concept of mixed states may be unfamiliar to many psychiatrists, recognition of these states may aid in the treatment of complex and/or treatment-refractory cases. In particular, mixed depression or depressive mixed states are common in a broad range of clinical settings. Furthermore, it is important to detect mixed depression as early as possible because pharmacological strategies differ between mixed and non-mixed depression. In this report, the author briefly reviews the clinical presentation, diagnosis, and impact of mixed depression on the course of mood disorders and pharmacological treatment.

\section{Definitions}

Various criteria are used to define mixed depression. Although most researchers use similar criteria for MDE (usually based on those outlined in the DSM-IIIR, IV, and IV-TR), the criteria for coexisting manic/hypomanic symptoms differ among researchers. Koukopoulos et al. placed emphasis on an excitatory state and the absence of inhibition [7], defining mixed depression as an MDE with $\geq 3$ of the following symptoms: racing or crowded thoughts, irritability or unprovoked feelings of rage, absence of psychomotor retardation, increased talkativeness, dramatic descriptions of suffering or frequent spells of weeping, mood lability, marked emotional reactivity, and early insomnia. In contrast, Perugi et al. defined mixed depression as an MDE with $\geq 3$ of the following 14 symptoms: irritable mood, emotional/mood lability, agitation, distractibility, psychomotor impulsivity, aggression (verbal or physical), racing thoughts, increased talkativeness/pressure to keep talking, hyperactivity, increased energy, risky behavior, grandiosity, elation, and hypersexuality [8]. Franco Benazzi, an Italian psychiatrist, defined mixed depression more simply as the co-existence of an MDE and $\geq 3$ of the following manic/hypomanic symptoms outlined in the DSM-IV: elevated mood, irritability, grandi- 
Table 1. Prevalence of manic/hypomanic symptoms during major depressive episodes

\begin{tabular}{lccc}
\hline & Total $(\mathrm{n}=217)$ & MDD $(\mathrm{n}=125)$ & BP-II/BP-NOS $(\mathrm{n}=92)$ \\
\cline { 2 - 4 } & & $\mathrm{n}(\%)$ & \\
\hline Manic/hypomanic symptoms & $116(53.5)$ & $61(48.8)$ & $55(59.8)$ \\
Psychomotor agitation & $79(36.4)$ & $25(20.0)$ & $54(58.7)$ \\
Racing/crowded thoughts & $78(35.9)$ & $19(15.2)$ & $59(64.1)$ \\
Irritability & $55(25.3)$ & $18(14.4)$ & $37(40.2)$ \\
Distractibility & $48(22.1)$ & $19(15.2)$ & $29(31.5)$ \\
Increased talkativeness & $25(11.5)$ & $9(7.2)$ & $16(17.9)$ \\
Increased goal-directed activity & $18(8.3)$ & $2(1.6)$ & $8(8.7)$ \\
Risky behavior & $11(5.1)$ & $3(2.4)$ & $1(1.1)$ \\
Decreased need for sleep & $1(0.5)$ & $0(0.0)$ & $0(0.0)$ \\
Inflated self-esteem & $1(0.5)$ & $1(0.8)$ & \\
Elevated mood & Tak & & 16 \\
\hline
\end{tabular}

This table is adapted from a 2015 report by Takeshima and Oka [10] (Psychiatry Clin Neurosci). Abbreviations: BP-II, bipolar II disorder; BP-NOS, bipolar disorder not otherwise specified; MDD, major depressive disorder.

osity, racing (or crowded) thoughts, distractibility, psychomotor agitation, increased talkativeness, hyperactivity, and risky behavior for $\geq 1$ week at the time of the interview [9]. While these diverse criteria have resulted in a wide range of prevalence rates among studies, researchers generally agree that mixed depression is associated with non-goaldirected excitatory symptoms such as irritability, racing/crowded thoughts, or psychomotor agitation [10]. In addition, previous studies have reported fairly consistent clinical characteristics, course, and prognosis (as described in a later section).

\section{Prevalence, symptomatology, and diagnostic issues}

In contrast to mixed mania, which exists only in patients with BD, mixed depression can be observed in both patients with $\mathrm{BD}$ and those with MDD. In this way, mixed depression does not necessarily reflect an admixture of manic/hypomanic and depressive symptoms, but rather represents a bridge between bipolar and monopolar illnesses. Mixed depression is common in clinical settings, accounting for $48.9 \%$ (median, range: $22.9-72.3 \%$, nine studies [7, 9-16]) of MDEs due to $\mathrm{BD}$ and $31 \%$ (median, range 12.8-39.7\%, seven studies [7, 9, 10, 13-16]) of MDEs due to MDD. In most cases, depressive, dysphoric, or irritable mood predominate, while elevated/expansive moods are more rarely observed. Frequently observed manic/hypomanic symptoms include distractibility, racing/ crowded thoughts, irritability, psychomotor agitation, and increased talkativeness (Table 1, adapted from Takeshima and Oka [10]). Patients with rac- ing/crowded thoughts experience thoughts at an abnormal speed or an increase in the amount of thoughts. Racing thoughts are those that occur in rapid succession or continuously overlap, without any link between them [17]. In case of crowded thoughts, the patient complains that his or her head is full of thoughts of all kinds [17]. The pain and/or distress of racing/crowded thoughts can lead to selfharm (i.e., wrist cutting, drug overdose). Although racing/crowded thoughts are among the most frequent symptoms in patients with mixed depression, they are rarely reported by patients spontaneously. Thus, clinicians should specifically inquire regarding these symptoms in patients with depression in order to establish the diagnosis of mixed depression. In addition, because racing/crowded thoughts are a risk factor for suicidality, clinicians should be particularly sensitive to these symptoms to reduce risk among patients with mixed depression.

Under-diagnosis of mixed depression is quite common. Most patients with mixed depression are diagnosed with "major depression." Given the available evidence, all patients should be assessed for the coexistence of manic/hypomanic symptoms at each visit. For example, patients can be screened using scales for depression (e.g., Montgomery-Åsberg Depression Rating Scale) and mania/hypomania (e.g., Young Mania Rating Scale) simultaneously. Because patients with mixed depression generally lack psychomotor retardation and often appear to exhibit mild depression, they also tend to be diagnosed with adjustment disorder. Diagnoses of anxiety disorders are likely in patients with psychomotor agitation, distractibility, and co-morbid symp- 
toms of anxiety. Such patients are also frequently diagnosed with borderline personality disorder due to the presence of prominent mood lability and emotional reactivity, anger-related outbursts, and self-harmful behaviors. However, in patients with borderline personality disorder, symptoms usually begin in adolescence or early adulthood and continue without remission, while mixed depression exhibits an episodic course.

\section{Impact on mood disorders}

Mixed depression strongly suggests a diagnosis of $\mathrm{BD}$ in patients experiencing an MDE. In previous studies [18, 19], the authors aimed to extract independent clinical features suggestive of BD diagnosis from a variety of clinical features, with the goal of developing a predictive model for BD diagnosis in patients experiencing an MDE. Among the 12 and 74 respective clinical features, mixed depression (defined as an MDE with $\geq 3$ non-overlapping or overlapping manic/hypomanic symptoms) remained one of five independent features that could predict BD diagnosis (odds ratios: 5.57 and 17.9, respectively) $[18,19]$. Furthermore, previous studies have reported that subthreshold manic/hypomanic symptoms are frequently associated with subsequent progression to $\mathrm{BD}$ in patients with MDD diagnoses. Associations have also been observed for antidepressant-induced mania/hypomania and a family history of BD, suggesting that mixed "monopolar" major depression belongs to the bipolar spectrum $[15,20,21]$.

Mixed depression is associated with relatively poorer long-term prognosis than non-mixed depression. Patients with mixed depression are more likely to experience mixed states during the first episode, experience more severe episodes of longer duration, and exhibit shorter inter-episodic remission periods than those without. In addition, patients with mixed depression exhibit higher recurrence rates, more rapid cycling, higher rates of comorbid substance use and anxiety disorders, and an increased risk of suicidal behaviors [5].

\section{DSM-5 definition including the "mixed features" specifier}

In the DSM-5, mixed depression corresponds to an MDE with mixed features, which is defined as an MDE with $\geq 3$ non-overlapping manic/hypomanic symptoms. Irritability, psychomotor agitation, and distractibility are excluded because these symptoms overlap with those of depression. However, some researchers have expressed concerns regarding this stipulation because overlapping symptoms are common and have even been emphasized as the core symptoms of mixed depression, as described elsewhere [10]. Several studies have noted that the prevalence of mixed depression is only $7.4 \%$ in patients with BD (median, range $6.3-17.5 \%$, four studies $[10-12,15])$ and $5.6 \%$ in patients with MDD (median, range 0-15.5\%, three studies [10, $15,22])$ when these criteria are adopted. Furthermore, more inclusive definitions of mixed depression (i.e., an MDE with $\geq 3$ non-overlapping or overlapping manic/hypomanic symptoms) have yielded statistically more robust associations with several illness-related characteristics of $\mathrm{BD}$ than DSM-5 criteria [8]. Thus, the sensitivity of the DSM-5 definition may be too low for detecting mixed depression in most patients. Therefore, the author recommends using more inclusive definitions of mixed depression, at least for clinical purposes.

\section{Pharmacotherapy for mixed depression}

Pharmacotherapy for mixed states is challenging because physicians are required to treat both manic/ hypomanic and depressive symptoms concurrently. Antidepressant use can exacerbate symptoms of agitation and irritability without improving depressive symptoms. In addition, such treatment can induce manic/hypomanic switch and/or so-called "activation syndrome," which may lead to newly developed suicidality [23, 24]. As such, the International Society for Bipolar Disorders Task-force Report on antidepressant use in BD recommends avoiding antidepressant use during manic and depressive episodes with mixed features and discontinuing previously prescribed antidepressants in patients currently experiencing mixed states [25].

As interest in mixed states has been revived only recently, few prospective clinical trials have focused on mixed states. Most evidence regarding appropriate pharmacotherapy is related to mixed mania/hypomania, and has been collected from post hoc subgroup and pooled analyses of data from randomized controlled trials (RCTs) for acute manic and mixed episodes in patients with BD-I [4]. Evidence regarding pharmacotherapy for mixed depression is even more scarce. Nonetheless, six international guidelines have mentioned pharmacological strategies for mixed depression [26]. The mainstay 
Table 2. Comparison of guidelines: evidence regarding the efficacy of acute treatment for mixed depression

\begin{tabular}{|c|c|c|c|c|}
\hline \multirow{2}{*}{ Guidelines } & \multicolumn{2}{|c|}{ First-line } & \multicolumn{2}{|c|}{ Second-line } \\
\hline & Monotherapy & Combination & Monotherapy & Combination \\
\hline BAB $3^{\text {rd }}$ Edition & - & - & - & - \\
\hline $\begin{array}{l}\text { CANMAT/ISBD } \\
2018\end{array}$ & SGA: LUR (2) & $\begin{array}{l}\mathrm{LUR}+\mathrm{Li} / \mathrm{VPA}{ }^{(1)} \\
\mathrm{OFC}^{(2)}\end{array}$ & - & $\cdot \mathrm{ASN}^{(4)}$ \\
\hline CINP-BD 2017 & - & $\mathrm{OLZ}+\mathrm{MS}^{(2)}$ & $\begin{array}{l}\text { SGA: ARP, ASN, } \\
\text { OLZ }{ }^{(3)}, \mathrm{ZPD}^{(4)} \\
{\mathrm{MS}: \mathrm{CBZ}^{(3)}, \mathrm{VPA}^{(4)}}^{\mathrm{OFC}^{(4)}}\end{array}$ & - \\
\hline $\begin{array}{l}\text { RANZCP Mood } \\
\text { Disorders CPG }\end{array}$ & $\begin{array}{l}\text { SGA: OLZ, QTP } \\
\text { MS: VPA }\end{array}$ & $\begin{array}{l}\text { SGA (OLZ, QTP) or } \\
\text { VPA + AD } \\
\text { OLZ + VPA }\end{array}$ & - & - \\
\hline $\begin{array}{l}\text { Mixed depression } \\
\text { guidelines }\end{array}$ & $\begin{array}{l}\text { SGA: LUR, ASN, } \\
\text { QTP, QTP-XR, ARP, } \\
\text { ZPD }{ }^{(1)}, \text { OLZ, CAR }{ }^{(2)} \\
\text { MS: LMT, VPA, Li }{ }^{(2)}\end{array}$ & $\begin{array}{l}\mathrm{SGA}+\mathrm{MS} \\
(\mathrm{Li} / \mathrm{LMT} / \mathrm{VPA}) \\
\mathrm{Li}+\mathrm{VPA} \\
\mathrm{Li} / \mathrm{VPA}+\mathrm{LMT} \\
\mathrm{OFC}\end{array}$ & MS: CBZ ${ }^{(3)}$ & $\begin{array}{l}\cdot \mathrm{CBZ}+\mathrm{Li}^{(3)} \\
\cdot \mathrm{Li}+\text { pramipexole }^{(3)} \\
\cdot \mathrm{Li}+\mathrm{ECT}^{(3)} \\
\cdot \mathrm{MS} \text { or } \mathrm{SGA}+\mathrm{SSRI} / \text { bupropion/ MAOI }\end{array}$ \\
\hline $\begin{array}{l}\text { WFSBP mixed } \\
\text { states }\end{array}$ & - & $\mathrm{ZPD}+\mathrm{TAU}$ & $\begin{array}{l}\text { SGA: LUR, OLZ } \\
\text { MS: CBZ }{ }^{(3)}\end{array}$ & $\cdot \mathrm{ECT}$ \\
\hline
\end{tabular}

This table is adapted from a 2018 report by Verdolini et al. [26] (Acta Psychiatr Scand). (1) - (4), grades of evidence; AD, antidepressants; ARP, aripiprazole; ASP, asenapine; CAR, cariprazine; ECT, electroconvulsive therapy; Li, lithium; LMT, lamotrigine; LUR, lurasidone; MAOI, monoamine oxidase inhibitor; MS, mood stabilizers; OFC, olanzapine + fluoxetine; OLZ, olanzapine; QTP, quetiapine; SGA, second-generation antipsychotics; SSRI, selective serotonin reuptake inhibitor; TAU, treatment as usual; VPA, valproic acid; ZPD, ziprasidone.

of acute-phase pharmacotherapy for mixed depression is treatment with second-generation antipsychotics (SGAs), either alone or in combination with mood stabilizers (Table 2, adapted from Verdolini et al. [26]). In particular, olanzapine, olanzapine plus fluoxetine, ziprasidone, and lurasidone have been proven effective in the treatment of acute-phase mixed depression in post hoc (the former two compounds) and prospective studies (the latter two compounds) [26]. Current guidelines indicate that these agents should be utilized alone or in combination with mood stabilizers as first-line pharmacotherapy for acute-phase mixed depression [26]. Although no trials have specifically investigated the efficacy of quetiapine for mixed depression, this agent is effective in treating both acute manic and depressive episodes. Thus, two guidelines ranked quetiapine as the most appropriate first-line treatment option for acute-phase mixed depression [26]. However, the most appropriate treatments for "monopolar" mixed depression (mixed depression due to MDD) remain to be elucidated. One RCT reported that monotherapy with lurasidone and addon ziprasidone are effective and well-tolerated in this patient population [27, 28]. Considering these results and the bipolar nature of monopolar mixed depression, it may be helpful to adopt similar pharmacological strategies for monopolar mixed depression and bipolar mixed depression.

To the best of the author's knowledge, no clinical trials have specifically investigated maintenance pharmacotherapy for mixed depression. One guideline recommends continuing agents that were efficacious in the acute phase (Figure 2, adapted from Stahl et al. [29]). Other guidelines recommend valproate, lithium, quetiapine, olanzapine, and a combination of quetiapine and lithium/valproate based on results derived from data obtained using the DSM-IV definition of mixed episode [26]. Despite a lack of relevant studies to date, combined use of lamotrigine and agents that were efficacious in the acute phase may represent an effective maintenance strategy, given the proven protective effect of lamotrigine against depressive relapse in patients with BD [30]. Current guidelines also recommend electroconvulsive therapy as an effective option in treatment-resistant patients [26]. All guidelines agree in interrupting antidepressant monotherapy or adding mood-stabilizing medications for patients with bipolar or monopolar mixed depression [26]. 


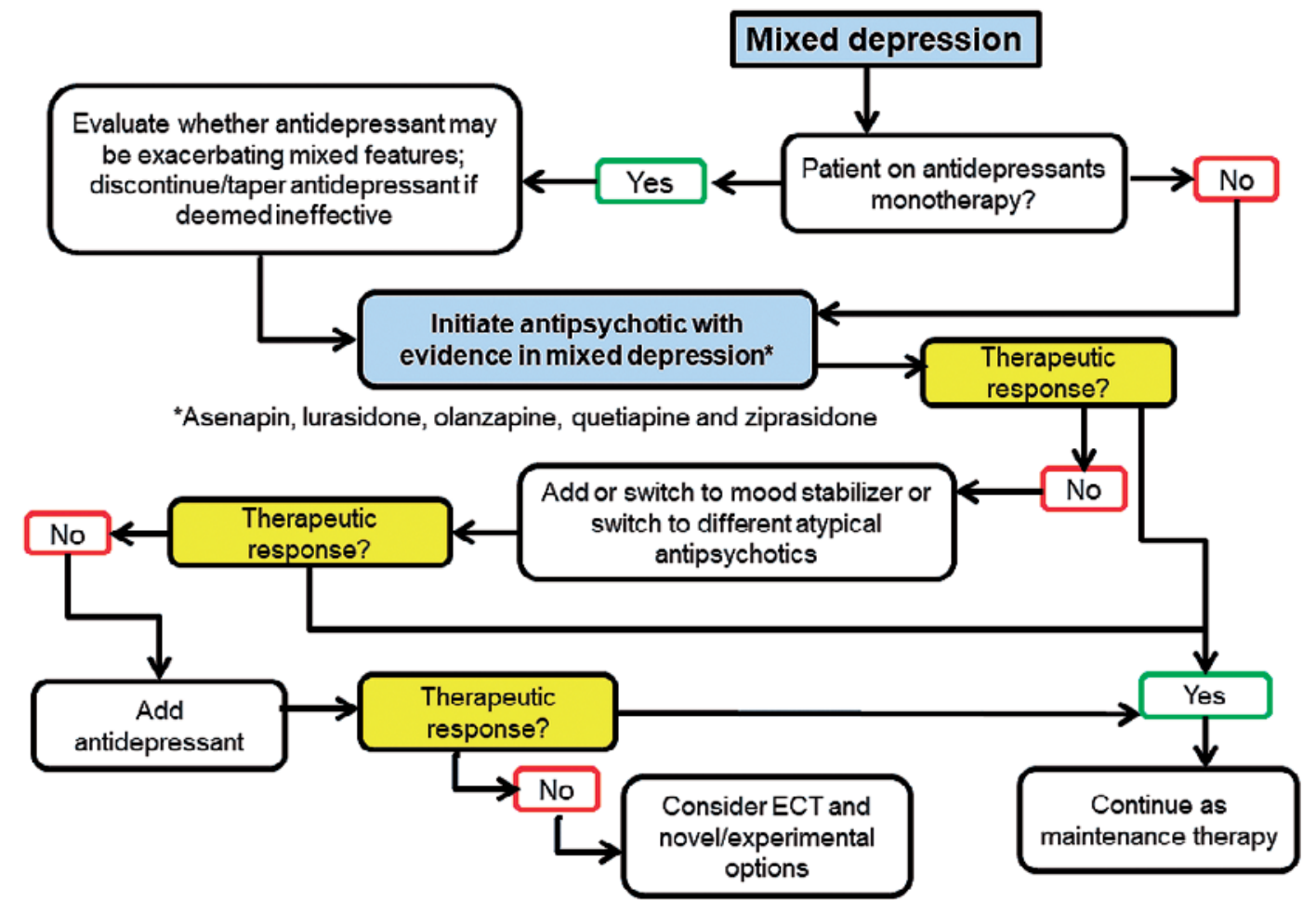

Figure 2. Treatment algorithm for mixed depression. This figure is adapted from a 2017 report by Stahl et al. [29] (CNS Spectr).

\section{Conclusion}

Mixed depression is a common yet underdiagnosed clinical entity, and pharmacological strategies are largely different for mixed and non-mixed depression. Indeed, in patients with mixed depression, antidepressant use may exacerbate agitation/irritability and induce newly developed suicidality without improving depressive symptoms. Pharmacological mainstays for mixed depression include several SGAs such as olanzapine, a combination of olanzapine and fluoxetine, ziprasidone, lurasidone, and quetiapine as well as mood stabilizers. Given the differences in pharmacological strategies for mixed versus non-mixed depression, patients with depression should be assessed for co-existing manic/hypomanic symptoms at each visit, in order to detect mixed depression as early as possible.

While several reviews have discussed mixed states, the present review focused on mixed depression, the issues of under- and misdiagnosis, and detailed symptomatology to facilitate early diagnosis. Furthermore, we discussed the latest findings concerning the validity of "mixed features" as specified by the DSM- 5 and recent international guidelines concerning pharmacotherapeutic strategies for mixed depression.
However, several issues remain to be resolved. A better understanding of the neurobiology of mixed depression is necessary for predicting treatment responses and developing more effective treatments. Because current data are largely dependent on post hoc subgroup analyses, additional prospective clinical trials are required to investigate acute-phase and maintenance pharmacotherapy for mixed depression. Furthermore, future studies should aim to determine the adequate dosage of SGAs for acutephase treatment, as well as the potential of lamotrigine to prevent relapse. Researchers should also investigate differences in clinical characteristics, treatment responses, and long-term course between patients with bipolar and monopolar mixed depression.

\section{Conflicts of interest}

The author has no conflicts of interest to declare.

\section{ACKNOWLEDGEMENT}

The author would like to thank Editage (www. editage.com) for English language editing. No financial support was provided for this review. 


\section{REFERENCES}

[1] Marneros A. Origin and development of concepts of bipolar mixed states. J Affect Disord 2001; 67: 229-240.

[2] Kraepelin E. Psychiatrie. Ein Lehrbuch fur Studierende und Arzte. III. Bd. Klinische Psychiatrie. II. Teil (8.Volstandigumgearbeitete Auflage). Leipzig: Johann Ambrosius Barth; 1913.

[3] American Psychiatric Association, editor. Diagnostic and Statistical Manual of Mental Disorders, Fourth Edition, Text Revision. Washington, DC: Author; 2000.

[4] Takeshima M. Treating mixed mania/hypomania: A review and synthesis of the evidence. CNS Spectr 2017; 22: 177-185.

[5] Swann AC, Lafer B, Perugi G, et al. Bipolar mixed states: An International Society for Bipolar Disorders task force report of symptom structure, course of illness, and diagnosis. Am J Psychiatry 2013; 170: 31-42.

[6] American Psychiatric Association, editor. Diagnostic and Statistical Manual of Mental Disorders, Fifth Edition. Arlington, VA: Author; 2013.

[7] Koukopoulos A, Sani G, Koukopoulos AE, et al. Melancholia agitata and mixed depression. Acta Psychiatr Scand Suppl 2007; 433: 50-57.

[8] Perugi G, Angst J, Azorin JM, et al. Mixed features in patients with a major depressive episode: The BRIDGE-II-MIX study. J Clin Psychiatry 2015; 76: e351-e358.

[9] Benazzi F. Defining mixed depression. Prog Neuropsychopharmacol Biol Psychiatry 2008; 32: 932-939.

[10] Takeshima M, Oka T. DSM-5-defined 'mixed features' and Benazzi's mixed depression: Which is practically useful to discriminate bipolar disorder from unipolar depression in patients with depression? Psychiatry Clin Neurosci 2015; 69: 109-116.

[11] Kim H, Kim W, Citrome L, et al. More inclusive bipolar mixed depression definition by permitting overlapping and non-overlapping mood elevation symptoms. Acta Psychiatr Scand 2016; 134: 199-206.

[12] Miller S, Suppes T, Mintz J, et al. Mixed depression in bipolar disorder: Prevalence rate and clinical correlates during naturalistic follow-up in the Stanley Bipolar Network. Am J Psychiatry 2016; 173: 1015-1023.

[13] Angst J, Azorin JM, Bowden CL, et al. Preva- lence and characteristics of undiagnosed bipolar disorders in patients with a major depressive episode: The BRIDGE study. Arch Gen Psychiatry 2011; 68: 791-798.

[14] Azorin JM, Kaladjian A, Adida M, et al. Selfassessment and characteristics of mixed depression in the French national EPIDEP study. J Affect Disord 2012; 143: 109-117.

[15] Barbuti M, Pacchiarotti I, Vieta E, et al. Antidepressant-induced hypomania/mania in patients with major depression: Evidence from the BRIDGE-II-MIX study. J Affect Disord 2017; 219: 187-192.

[16] Takeshima M, Kitamura T, Kitamura M, et al. Impact of depressive mixed state in an emergency psychiatry setting: A marker of bipolar disorder and a possible risk factor for emergency hospitalization. J Affect Disord 2008; 111: 52-60.

[17] Koukopoulos A, Koukopoulos A. Agitated depression as a mixed state and the problem of melancholia. Psychiatr Clin North Am 1999; 22: $547-564$.

[18] Takeshima M, Oka T. A comprehensive analysis of features that suggest bipolarity in patients with a major depressive episode: Which is the best combination to predict soft bipolarity diagnosis? J Affect Disord 2013; 147: 150155.

[19] Inoue T, Inagaki Y, Kimura T, et al. Prevalence and predictors of bipolar disorders in patients with a major depressive episode: The Japanese epidemiological trial with latest measure of bipolar disorder (JET-LMBP). J Affect Disord 2014; 174C: 535-541.

[20] Fiedorowicz JG, Endicott J, Leon AC, et al. Subthreshold hypomanic symptoms in progression from unipolar major depression to bipolar disorder. Am J Psychiatry 2011; 168: 40-48.

[21] Benazzi F. Mixed depression: A clinical marker of bipolar-II disorder. Prog Neuropsychopharmacol Biol Psychiatry 2005; 29: 267274.

[22] Hasin DS, Sarvet AL, Meyers JL, et al. Epidemiology of adult DSM-5 major depressive disorder and its specifiers in the United States. JAMA Psychiatry 2018; 75: 336-346.

[23] Goldberg JF, Perlis RH, Ghaemi SN, et al. Adjunctive antidepressant use and symptomatic recovery among bipolar depressed patients with concomitant manic symptoms: Findings from the STEP-BD. Am J Psychiatry 2007; 164: 1348-1355. 
[24] Takeshima M, Oka T. Association between the so-called "activation syndrome" and bipolar II disorder, a related disorder, and bipolar suggestive features in outpatients with depression. J Affect Disord 2013; 151: 196-202.

[25] Pacchiarotti I, Bond DJ, Baldessarini RJ, et al. The International Society for Bipolar Disorders (ISBD) task force report on antidepressant use in bipolar disorders. Am J Psychiatry 2013; 170: 1249-1262.

[26] Verdolini N, Hidalgo-Mazzei D, Murru A, et al. Mixed states in bipolar and major depressive disorders: Systematic review and quality appraisal of guidelines. Acta Psychiatr Scand 2018; 138: 196-222.

[27] Suppes T, Silva R, Cucchiaro J, et al. Lurasidone for the treatment of major depressive dis- order with mixed features: A randomized, double-blind, placebo-controlled study. Am J Psychiatry 2016; 173: 400-407.

[28] Patkar A, Gilmer W, Pae CU, et al. A 6 week randomized double-blind placebo-controlled trial of ziprasidone for the acute depressive mixed state. PLoS One 2012; 7: e34757.

[29] Stahl SM, Morrissette DA, Faedda G, et al. Guidelines for the recognition and management of mixed depression. CNS Spectr 2017; 22: 203-219.

[30] Goodwin GM, Bowden CL, Calabrese JR, et al. A pooled analysis of 2 placebo-controlled 18-month trials of lamotrigine and lithium maintenance in bipolar I disorder. J Clin Psychiatry 2004 ; 65 : 432-441. 